\title{
Proprioceptive Neuromuscular Facilitation in Shoulder Rehabilitation of Women Submitted to Surgical Treatment for Breast Cancer
}

\author{
Elaine Caldeira de Oliveira Guirro ${ }^{1,2, *}$, Daniela Santana Polati Silveira ${ }^{2}$, Carla Silva Perez ${ }^{2}$, Thais Montezuma ${ }^{2}$, Monique Silva \\ Rezende $^{2}$, Raul Oliveira ${ }^{3}$ and Rinaldo Roberto de Jesus Guirro ${ }^{1,2}$ \\ ${ }^{1}$ Department of Health Sciences, Ribeirão Preto Medical School, University of São Paulo, Ribeirão Preto-SP, Brazil \\ ${ }^{2}$ Postgraduate Program in RehabilitationandFunctional Performance, São Paulo University, Ribeirão Preto, São Paulo, Brazil \\ ${ }^{3}$ CIPER, Neuromuscular ResearchLaboratory, Universidade de Lisboa, Lisboa, 1649-004, Portugal
}

\section{Abstract}

Background: Survivors of breast cancer show loss of function of the homolateral limb after treatment, which involves reduction of the functional range of the shoulder, as well as impairment of the involved musculature. The aim of this study was to evaluate the electromyographic activity and the strength of the muscles in the shoulder complex of women who have been subjected to mastectomies, after an intervention using Proprioceptive Neuromuscular Facilitation (PNF) exercises.

Methods: This study included 18 women submitted to modified radical mastectomy, with a mean age of $52.55 \pm 8.42$ years. The training exercises with Proprioceptive Neuromuscular Facilitation occurred two times per week for eight weeks, the standards used were the diagonal pattern, with flexion-abductionexternal rotation and extension-adduction-internal rotation. For the evaluation of the electromyographic activity, electrodes were positioned in the muscles of the upper trapezius and deltoid (anterior, middle and posterior muscle fibers).

Results: The electromyographic analysis demonstrated a 32\% elevation in the Standard Envelope (SE) of the middle fiber of the deltoid muscle (DM), $15 \%$ in the SE of the upper trapezius (UT), an increase in muscle strength of $15 \%$ in the DM and $15 \%$ in the UT, also raising the strength of these muscles by $36 \%$.

Conclusion: The results of the proposed intervention allow us to conclude that, for the sample evaluated, there was an increase in the recruitment of motor units and of muscle strength of the shoulder affected by the surgery to treat breast cancer.

\section{Introduction}

It is challenging to acquire knowledge on the functional changes of the upper limb and shoulder due to breast cancer treatment, such as reduction of movement, muscle weakness, pain, numbness, and lymphedema, because these changes causes numerous chronic impacts, with a clinically significant decrease in the functional capacity and quality of life of these women [1-3].

Functional limitations associated with pain and the decrease of shoulder movement can affect daily and recreational activities of women submitted to breast cancer treatments [4], besides promoting 2.5 times more chances of losing some productive capacity at work, generating higher financial costs [5].

From six to twelve months after treatment, at least half of the women reported a decrease in the range of motion of the upper limb affected. The movements of abduction and external rotation of the shoulder were the most affected [6].

Kinematic parameters and muscle EMG of the shoulder waist muscles and upper limbs are altered in survivors of breast cancer, but patients submitted to mastectomy demonstrate larger deviations of movement and higher levels of pain, symptoms which are more chronic when compared to the other surgeries [7]. The rhomboids and upper trapezius muscles show the greatest loss of electromyographic activity in short and long terms, and this loss is associated with increased disabling pain [8].

The technique of Proprioceptive Neuromuscular Facilitation (PNF) is designed to promote or accelerate the response of the neuromuscular mechanism through stimulation of the proprioceptors. It is a pattern of muscle contractions and diagonal movements that increase active movements, produce a correct direction of movement, and reduces fatigue, besidespromotingmuscularstrengtheningandstretching $[9,10]$.

The PNF demonstrates good results in the gain of amplitude and decrease of pain in the frozen shoulder syndrome [11], in the gain of movement in the adhesive capsulitis of the shoulder [12], and in the gain of stretching of the shoulder musculature in athletes [13]. However, the use of this technique in the rehabilitation of breast cancer survivors is still very scarce in the literature.

Shoulder muscle rehabilitation in breast cancer survivors has an effect on the improvement of functional welfare, and has the potential to attenuate the negative impacts that the morbidities of the upper limb cause to the emotional health of these women [14].

More appropriate rehabilitation programs are needed to deal specifically with the muscles involved in the function of the shoulder

"Corresponding Author: Dr. Elaine Caldeira de Oliveira Guirro, Universidade de São Paulo, Ribeirão Preto, SãoPaulo, Brazil, Bandeirantes Avenue, 3900, Ribeirão Preto, São Paulo 14049900, Brazil, Tel: +5516 3315-4584; E-mail: ecguirro@fmrp.usp.br

Citation: Guirro ECO, Silveira DSP, Perez CS, Montezuma T, Rezende MS, et al. (2019) Proprioceptive Neuromuscular Facilitation in Shoulder Rehabilitation of Women Submitted to Surgical Treatment for Breast Cancer. Int J Phys Ther Rehab 5: 155. doi: https://doi.org/10.15344/2455-7498/2019/155

Copyright: (C) 2019 Guirro et al. This is an open-access article distributed under the terms of the Creative Commons Attribution License, which permits unrestricted use, distribution, and reproduction in any medium, provided the original author and source are credited. 
Citation: Guirro ECO, Silveira DSP, Perez CS, Montezuma T, Rezende MS, et al. (2019) Proprioceptive Neuromuscular Facilitation in Shoulder Rehabilitation of Women Submitted to Surgical Treatment for Breast Cancer. Int J Phys Ther Rehab 5: 155. doi: https://doi.org/10.15344/2455-7498/2019/155

Page 2 of 6

that is homolateral to the surgical procedure done to breast cancer survivors. The aim of this study was to evaluate the electromyographic activity and the strength of the muscles in the shoulder complex of women who have been subjected to mastectomies, after an intervention using Proprioceptive Neuromuscular Facilitation (PNF) exercises.

\section{Methods}

\section{Subjects}

Eighteen women were selected, who had been submitted to the surgery of modified radical mastectomy with axillary lymphadenectomy, with a mean age of $52.55 \pm 8.42$ years, who were irregularly physically active according to the criteria established by the International Physical Activity Questionnaire (IPAQ).

The subjects were submitted to a standard anamnesis, and the following traits were considered as exclusion criteria: trauma in the region of the shoulder and shoulder waist muscles, presence of clinical signs of instability of the shoulder joint, women already undergoing physical therapy treatments, and those who presented lymphedema.

\section{Ethical approval}

All volunteers signed a consent form, the procedures of the study were approved by the Research Ethics Committee of the Clinical Hospital of the Faculty of Medicine of Ribeirão Preto, at the Universidade de São Paulo, under protocol number 2890/2017.

\section{Protocol of electromyography}

For the electromyographic record, were used: 1) Signal Acquisition System - Lynx Electronics Ltda ${ }^{\circ}$. Signal Conditioner Module With 16 channels; 2) 12-bit converter board A/D CAD model 12/32 from Lynx Electronics Ltda ${ }^{\star}$; 3) Aqdados Software version 4.6; 4) Simple differential active surface Electrodes from Lynx ${ }^{\circledR}$ Electronics Ltd., 5) Reference electrode; 6) Load Cell Model MM-100 from Kratos Dinamometers Ltda ${ }^{\circ}$; 7) Electrogoniometer to identify the positioning and angulation of the limb analyzed.

For the evaluation of the electromyographic activity of the trapezius (upper fibers) and deltoid (anterior, middle, and posterior muscle fibers), the electrodes were placed on standardized points on the skin according to the EMG Surface recommendations for the NonInvasive Assessment of Muscles SENIAM, and the signals were collected during maximum isometric contraction in abduction of $90^{\circ}$ of the upper limb in a neutral position regarding the rotation of the humeral scapula, for a period of five seconds.

The surface electrodes were placed over the motor points upper trapezius and middle deltoid of the homolateral upper limb, and connected to the channels of the electromyography, after being located by the TENS (Orion Tens) equipment. The electrodes were fixed with adhesive tape perpendicular to the direction of the fibers, as proposed by De Luca (1997).

The ground electrode was positioned with electroconductive gel and fixed by adhesive tape in the lateral epicondyle of the humerus of the limb to be analyzed. Before the start of the evaluation, the electromyography channels were calibrated according to the manual provided by the equipment manufacturer.

The load cell was attached to a metal device (made for this purpose) attached at one end to an arm brace, while the other, perpendicular, was attached to the ground, trough a steel chain. Thus, with the muscle contraction, the load cell was tensioned, and the reading of the torque was performed through observation of the monitor of the computer. The examined limb of the volunteer was positioned parallel to a support device, with the purpose to avoid fatigue.

\section{Intervention}

The protocol for muscle training consisted of exercises of Proprioceptive Neuromuscular Facilitation (PNF), in which the movement of the upper limb homolateral to the surgery was resisted manually by the same researcher, that was had experience with PNF.

The intervention was applied two times a week, over the course of eight weeks, the exercises followed in accordance to Wicke et al. (2014), aiming at stretching the muscles adjacent to the shoulder, as well as the training and gain of muscle strength. The standards used were the diagonal pattern, with flexion-abduction-external rotation and extension-adduction-internal rotation, performed with the elbow extended.

The volunteers remained in supine position with their hip joint flexed in $30^{\circ}$, and the lower limbs supported in semiflexion.

\section{Statistical analysis}

The sample was calculated through the Ene software, version 3.0 (Autonomous University of Barcelona, Barcelona, Spain). The sample size was calculated based on the study conducted by Shamley et al. (2007). Thus, the outcome variable chosen was the maximum speed of the arterial blood flow. In this context, considering a statistical power of $80 \%$ and alpha value of 0.05 , a number of 18 patients per group was estimated.

The statistical analysis of the data was performed after the completion of the entire experimental phase. Initially, it an exploratory analysis of the data was performed by the SAS - JMP software (Statistical Analysis System), with the normality test of Shapiro-Wilk for the statistical variable spectral density power $(n<2000)$, and the normality test KSL for strength and normalized envelope $(n>2000)$ of the different experimental groups. The Signed tests of Wilcoxon/ Kruskal Wallis were applied.

\section{Results}

The data for the envelope (Figure 1) normalized by the average amplitude of the electromyographic signal, showed a significant increase of $32 \%(p<0.0001)$ for the middle deltoid muscle fibers after physical therapy intervention, when compared to the values of the group before intervention (1), indicating an improvement in the function of this muscle.

Comparing the mean values before and after physical therapy intervention in Figure 2, significant increase of $15 \%(p<0.0001)$ can be observed in the median frequency of middle deltoid muscle fibers, after (2) intervention, indicating an increase in the recruitment of the motor units.

Figure 3 shows that there was a significant increase in the normalized envelope of the trapezius muscle of $15 \%(\mathrm{p}, 0,0001)$ after (2) therapeutic intervention, when compared to the results before (1) intervention, indicating an increase in the recruitment of the motor units. 
Citation: Guirro ECO, Silveira DSP, Perez CS, Montezuma T, Rezende MS, et al. (2019) Proprioceptive Neuromuscular Facilitation in Shoulder Rehabilitation of Women Submitted to Surgical Treatment for Breast Cancer. Int J Phys Ther Rehab 5: 155. doi: https://doi.org/10.15344/2455-7498/2019/155

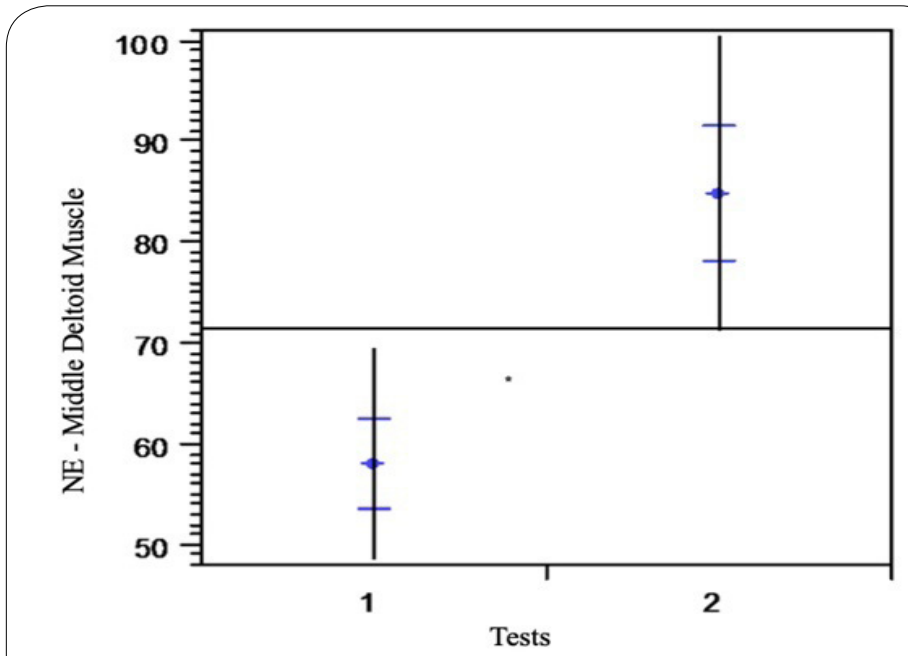

Figure 1: Mean \pm SD of the normalized envelope (NE) of the middle deltoid muscle fibers during maximum isometric contraction in the position of $90^{\circ}$ of arm abduction (1) before and (2) after physical therapy intervention.

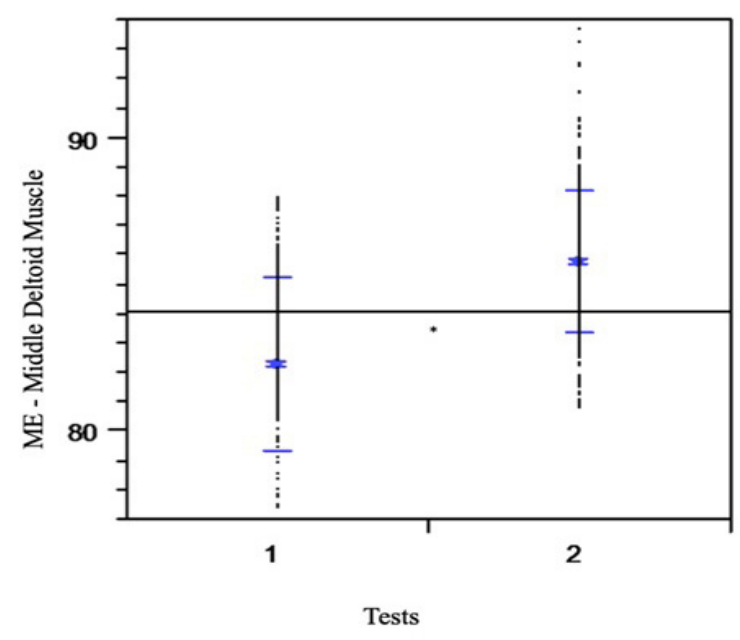

Figure 2: Mean \pm SD of median frequency (MF) of the middle deltoid muscle fibers during maximum isometric contraction in the position of $90^{\circ}$ arm abduction (1) before and (2) after physical therapy intervention.

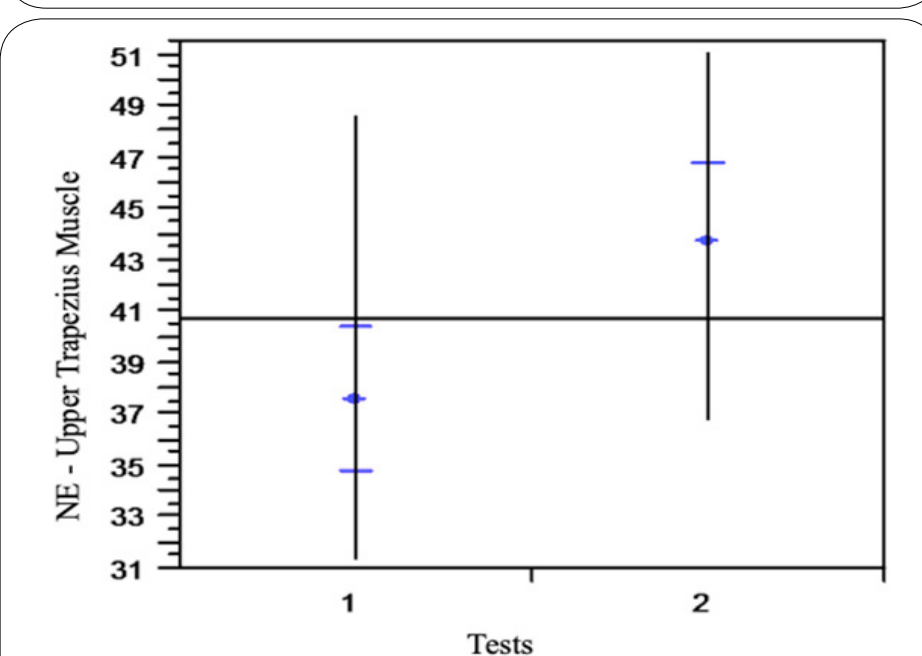

Figure 3: Mean \pm SD of the normalized envelope (NE) of the upper trapezius muscle during maximum isometric contraction in the position of $90^{\circ} \mathrm{arm}$ abduction (1) before and (2) after physical therapy intervention. 
Citation: Guirro ECO, Silveira DSP, Perez CS, Montezuma T, Rezende MS, et al. (2019) Proprioceptive Neuromuscular Facilitation in Shoulder Rehabilitation of Women Submitted to Surgical Treatment for Breast Cancer. Int J Phys Ther Rehab 5: 155. doi: https://doi.org/10.15344/2455-7498/2019/155

Page 4 of 6

The results of Figure 4 demonstrate that there has been a significant increase in the median frequency of the upper trapezius muscle in $4 \%(\mathrm{p}<0.0001)$ after $(2)$ intervention, when compared to the values of the group before intervention (1), indicating an improvement in the function of this muscle.

Figure 5 shows a significant increase, of $36 \%(\mathrm{p}<0.0001)$, of the strength after (2) physical therapy intervention, when compared to the results before it (1).

\section{Discussion}

In the present study it was observed that according to the envelope normalized by the average amplitude of the electromyographic signal, there was an increase of $32 \%(\mathrm{p}<0.0001)$ for deltoid muscle medium fibers and $15 \%(p<0.0001)$ in the median frequency after physical intervention, indicating an increase in motor unit recruitment.
There was a significant increase in the normal trapezius muscle envelope of $15 \%(\mathrm{p}<0.0001)$ and its median frequency in the upper trapezius muscle $4 \%(\mathrm{p}<0.0001)$ resulting in a significant increase in force during maximal isometric contraction in abduction. $90^{\circ}, 36 \%$ ( $\mathrm{p}$ $<0.0001$ ) after physical therapy intervention, evidencing the significant improvement in the increment of the motor units. The upper limb musculature of mastectomized women have higher levels of muscle effort on the affected side, however, weaknesses in the strength test, thus indicating a rehabilitation focused on strengthening exercises of this musculature [17].

The study of Zhu et al. (2016), considers the movement of the shoulder through physical activity a modifiable risk factor for primary and recurrent cancer, therefore, the need for rehabilitation in oncology becomes clear. Movement deviation patterns in women following surgery for breast cancer are similar to those seen in other known shoulder dysfunction. Exercise therapy and scapular stabilization

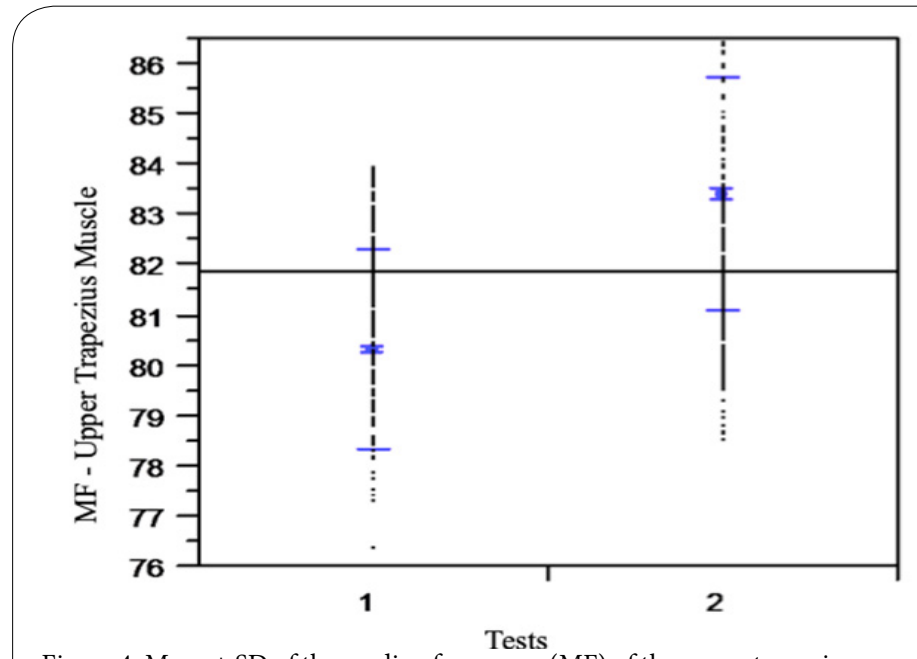

Figure 4: Mean \pm SD of the median frequency (MF) of the upper trapezius muscle during maximum isometric contraction in the position of $90^{\circ} \mathrm{arm}$ abduction (1) before and (2) after physical therapy intervention.

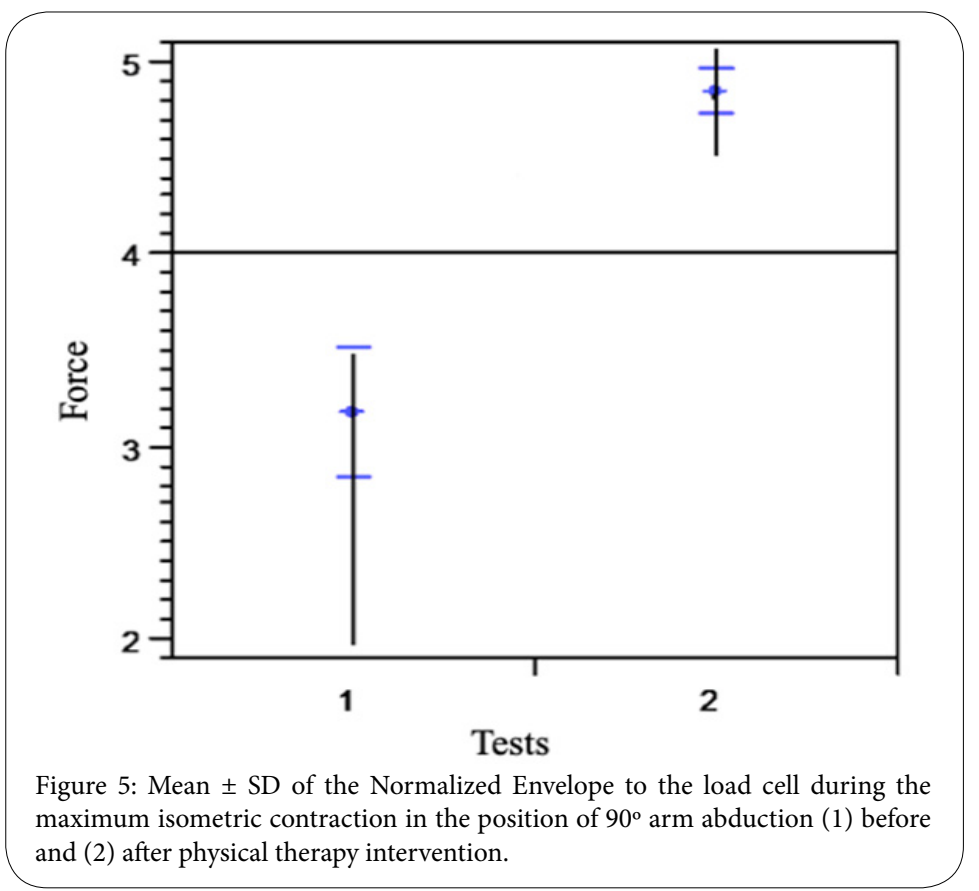


Citation: Guirro ECO, Silveira DSP, Perez CS, Montezuma T, Rezende MS, et al. (2019) Proprioceptive Neuromuscular Facilitation in Shoulder Rehabilitation of Women Submitted to Surgical Treatment for Breast Cancer. Int J Phys Ther Rehab 5: 155. doi: https://doi.org/10.15344/2455-7498/2019/155

Page 5 of 6

exercises were found to be an effective approach for controlling pain, promote normal motor control and decreasing disability [19].

The main sequelae in the postoperative period of modified radical mastectomy are changes in posture and in range of motion of the shoulder and reduction of muscular force homolateral to the surgery. Oxidative stress was also observed with a reduction of mitochondrial function and consequent alteration of physical capacity and physiological reserve. Thus, specific rehabilitation is needed during oncological treatment, aiming at functional independence and reinsertion of the individual in the society $[20,21]$.

It is necessary to establish an orientation program about the benefits of rehabilitation with exercises aimed at explaining doubts about cancer treatment, the practice of physical activity and improvement of the functional capacity of the patients. In addition, it is also necessary to explain the need to increase blood and lymphatic flow through muscle contraction, compensating the deleterious effects of the cancer treatment and its impact on the quality of life of oncological patients, preventing possible complications $[22,23]$.

Physical therapy has been pointed out as essential for the rehabilitation of mastectomized women, through the insertion of early interventions in the rehabilitation of cancer patients using the therapeutic arsenal that involves clinical practice, leading to improvements in their quality of life [24]. In this study, it was possible to find that the physical therapy intervention provided an increase in range of motion, muscle strength, and a significant reduction in pain in the shoulder joint, providing an improvement of functionality.

The PNF has been used in the rehabilitation of mastectomized women with the objective of increasing the lymphatic circulation of women with lymphedema. The biomechanical factors of the technique were observed in the study, which the method promoted a greater recruitment of the dynamic shoulder stabilizer muscles during the diagonal lift exercises, being pointed as an effective technique for the prevention, treatment and rehabilitation of shoulder disorders [25].

The benefits of proprioceptive neuromuscular facilitation in the gain of stretching and movement range of the shoulder joint can be observed. They improve functional performance due to the contraction of the antagonist during their execution, and the action of the professional facilitating the realization of the movement arc of the shoulder joint, which gives more credibility to the findings of this study [16], highlighting the findings of this study.

Increased activation of muscle fibers effectively improves functional autonomy and consequently activities of daily living, being crucial new rehabilitation techniques that favor the movements of the upper limb homolaterally [26]. Studies demonstrate the use of electromyography in the functional evaluation of patients submitted to mastectomy that evolved with functional changes of the limb homolateral to the surgery, which compromises the evolution of the functionality and promote a negative change in the quality of life, thus justifying its use in this study [27], thus enabling its use in the dear study.

During the execution of the electromyography tests, all necessary procedures for standardization were considered. Prior to the treatment of the data, the signals were individually analyzed trough sampling. The results demonstrated that the electromyographic circuit presented itself within normal standards, the distribution of the signal follows the Gaussian curve, and the number of bits were within the recommended $[28,29]$.
Aiming to clarify the communication of the central nervous system with the skeletal muscle system, the electromyography has been widely used in researches for analysis of human movement, being cited as an effective method to identify the activity of muscle contraction in the shoulder complex after the use of proprioceptive neuromuscular facilitation.

The findings of this study are limited to the detection of dysfunction resulting from a unilateral mastectomy. It would also be of interest to evaluate women who had undergone others types of surgery for the treatment.

Muscle electrical activity does not give information concerning muscle strength or the type of contractions that occur. However, the addition of force transducers and electrogoniometers, or some other type of measuring device to the recording system, allows for the simultaneous documentation of such data. This kind of record was extremely useful in the evaluation of the proposal.

\section{Conclusion}

From the results observed in the sample, it is possible to conclude that the therapeutic intervention, with the proprioceptive neuromuscular facilitation, increased in electromyographic activity and the strength of the muscles in the shoulder complex of women who have been subjected to mastectomies.

\section{Funding}

This study was financed in part by the Coordenação de Aperfeiçoamento de Pessoal de Nível Superior - Brasil (CAPES) Finance Code 88881.190398/2018-01.

\section{Competing Interests}

The authors declare that they have no competing interests.

\section{References}

1. Rietman J, Dijkstra PU, Hoekstra HJ, Eisma WH, Szabo BG, et al. (2003) Late morbidity after treatment of breast cancer in relation to daily activities and quality of life: a systematic review. Eur J Surg Oncol 29: 229-238.

2. Sagen $\AA$, Kåresen $R$, Sandvik L, Risberg MA (2009) Changes in arm morbidities and health-related quality of life after breast cancer surgery-a five-year follow-up study. Acta Oncol 48: 1111-1118.

3. Miaskowski C, Cooper B, Paul SM, West C, Langford D, et al. (2012) Identification of patient subgroups and risk factors for persistent breast pain following breast cancer surgery. J Pain 13:1172-1187.

4. Miedema $B$, Hamilton $R$ Tatemichi $S$, Thomas-MacLean $R$, Hack TF, et al. (2011) Do breast cancer survivors' post-surgery difficulties with recreational activities persist over time? Journal of Cancer Survivorship 5: 405-412.

5. Quinlan E, Thomas-MacLean R, Hack T, Kwan W, Miedema B, et al. (2009) The impact of breast cancer among Canadian women: disability and productivity. Work 34: 285-296.

6. Boquiren VM, Hack TF, Thomas RL, Towers A, Kwan WB, et al. (2016) A longitudinal analysis of chronic arm morbidity following breast cancer surgery. Breast Cancer Res Treat 157: 413-425.

7. Shamley D, Lascurain-Aguirrebeña I, Oskrochi R, Srinaganathan R (2012) Shoulder morbidity after treatment for breast cancer is bilateral and greater after mastectomy. Acta Oncol 51: 1045-1053.

8. Shamley DR, Srinanaganathan R, Weatherall R, Oskrochi R, Watson M, et al (2007) Changes in shoulder muscle size and activity following treatment for breast cancer. Breast Cancer Res Treat 106: 19-27. 
Citation: Guirro ECO, Silveira DSP, Perez CS, Montezuma T, Rezende MS, et al. (2019) Proprioceptive Neuromuscular Facilitation in Shoulder Rehabilitation of Women Submitted to Surgical Treatment for Breast Cancer. Int J Phys Ther Rehab 5: 155. doi: https://doi.org/10.15344/2455-7498/2019/155

9. Marek SM, Cramer JT, Fincher AL, Massey LL, Dangelmaier SM, et al. (2005) Acute effects of static and proprioceptive neuromuscular facilitation stretching on muscle strength and power output. J Athl Train 40: 94.

10. Lim W (2018) Optimal intensity of PNF stretching: maintaining the efficacy of stretching while ensuring its safety. J Phys Ther Sci 30: 1108-1111.

11. Lee BK (2015) Effects of the combined PNF and deep breathing exercises on the ROM and the VAS score of a frozen shoulder patient: Single case study. J Exerc Rehabil 11: 276

12. Balcı NC, Yuruk ZO, Zeybek A, Gulsen M, Tekindal MA, et al. (2016) Acute effect of scapular proprioceptive neuromuscular facilitation (PNF) techniques and classic exercises in adhesive capsulitis: a randomized controlled trial. J Phys Ther Sci 28: 1219-1227.

13. Decicco PV, Fisher MM (2005) The effects of proprioceptive neuromuscula facilitation stretching on shoulder range of motion in overhand athletes. Sports Med Phys Fitness 45: 183-187.

14. Boquiren VM, Hack TF, Thomas RL, Towers A, Kwan WB, et al. (2016) A longitudinal analysis of chronic arm morbidity following breast cancer surgery. Breast Cancer Res Treat 157: 413-425.

15. De Luca CJ (1997) The use of surface electromyography in biomechanics. Journal of applied biomechanics 13: 135-163.

16. Wicke J, Gainey K, Figueroa M (2014) A comparison of self-administered proprioceptive neuromuscular facilitation to static stretching on range of motion and flexibility. J Strength Cond Res 28: 168-172.

17. Brookham RL, Cudlip AC, Dickerson CR (2018) Quantification of upper limb electromyographic measures and dysfunction of breast cancer survivors during performance of functional dynamic tasks. Clin Biomech 52: 7-13.

18. Zhu G, Zhang $X$, Wang $Y$, Xiong $H$, Zhao $Y$, et al. (2016) Effects of exercise intervention in breast cancer survivors: a meta-analysis of 33 randomized controlled trails. Onco Targets Ther 9: 2153-2168.

19. Neto CM, Pezarat P, Oliveira R (2018) Effects of Breast Cancer Treatment on Shoulder Function: What to Expect and How to Treat? Int J Phys Ther Rehab 4: 147.

20. Rangel J, Perez CS, Oliveira R, Guirro ECO (2019) Effects of Breast Cancer Treatment on Posture: A Current Review. Int J Phys Ther Rehab 5: 152.

21. Loughney L, West MA, Kemp GJ, Grocott MPW, Jack S, et al. (2016) Exercise intervention in people with cancer undergoing neoadjuvant cancer treatment and surgery: a systematic review. Eur J Surg Oncol 42: 28-38.

22. Nelson NL (2016) Breast cancer-related lymphedema and resistance exercise: a systematic review. J Strength Cond Res 30: 2656-2665.

23. Cho Y, Do J, Jung S, Kwon O, Jeon JY, et al. (2016) Effects of a physical therapy program combined with manual lymphatic drainage on shoulde function, quality of life, lymphedema incidence, and pain in breast cancer patients with axillary web syndrome following axillary dissection. Support Care Cancer 24: 2047-2057.

24. Loh SY, Musa AN (2015) Methods to improve rehabilitation of patient following breast cancer surgery: a review of systematic reviews. Breast Cancer 7: 81

25. Comel JC, Nery RM, Garcia EL, da Silva Bueno C, de Oliveira SE, et al. (2018) A comparative study on the recruitment of shoulder stabilizing muscles and types of exercises. J Exerc Rehabil 26: 219-225.

26. Brookham RL, Dickerson CR (2016) Comparison of humeral rotation coactivation of breast cancer population and healthy shoulders. J Electromyog Kinesiol 29: 100-106.

27. Oskrochi G, Lesaffre E, Oskrochi Y, Shamley D (2016) An application of the multivariate linear mixed model to the analysis of shoulder complexity in Breast cancer patients. Int J Environ Res Public Health 1: 274.

28. Huang HY, Lin JJ, Guo YL, Wang WTJ, Chen YJ, et al. (2013) EMG biofeedback effectiveness to alter muscle activity pattern and scapular kinematics in subjects with and without shoulder impingement. J Electromyogr Kinesio 23: $267-274$

29. Jenkins ND, Housh TJ, Bergstrom HC, Cochrane KC, Hill EC, et al. (2016) Basic reporting and interpretation of surface EMG amplitude and mean power frequency: a reply to Vitgotsky, Ogborn, and Phillips Eur J App Physiol 116: 659-661. 